\title{
Lifetime Against Sublimation and an Initial Mass Estimate for the Exoplanet $\alpha$ Centauri Bb
}

\author{
Martin Beech ${ }^{1,2}$, Lowell Peltier ${ }^{2}$ \\ ${ }^{1}$ Campion College, the University of Regina, Regina, SK., Canada \\ ${ }^{2}$ Department of Physics, the University of Regina, Regina, SK., Canada \\ Email address: \\ beechm@uregina.ca (M. Beech), Peltier21@uregina.ca (L. Peltier)
}

\section{To cite this article:}

Martin Beech, Lowell Peltier. Lifetime Against Sublimation and an Initial Mass Estimate for the Exoplanet $\alpha$ Centauri Bb. American Journal of Astronomy and Astrophysics. Vol. 3, No. 4, 2015, pp. 70-76. doi: 10.11648/j.ajaa.20150304.11

\begin{abstract}
A two-component, core-mantle, model is developed to estimate the lifetime against destruction via sublimation of close-orbit, terrestrial-mass exoplanets. We specifically focus on the nearest terrestrial exoplanet, $\alpha$ Centauri $\mathrm{Bb}$, since the parent star $\alpha$ Cen B has a reasonably well determined age of $6 \pm 1$ Gyr. This latter knowledge specifically enables an estimate to be made of the amount of mantle material lost by $\alpha \mathrm{Cen} \mathrm{Bb}$ since the system formed. Our planet model allows for an ironcore and olivine mantle structure, and it also follows the luminosity evolution of $\alpha$ Cen B. Our results suggest that $\alpha \mathrm{Cen} \mathrm{Bb}$ had an initial mass of order $2 \mathrm{M}_{\text {Earth }}$, and that of order $0.2 \mathrm{M}_{\text {Earth }}$ of mantle material has been lost through sublimation since the planet formed. We additionally consider the fate of any putative planets, moving on circular orbits, interior to $\alpha \mathrm{Cen} \mathrm{Bb}$ (which has an orbital radius of $0.04 \mathrm{au}$ ), and it is found that any Earth mass, or lesser objects, orbiting closer than 0.024 au to $\alpha$ Cen B have lifetimes against destruction by sublimation smaller than 5 billion years.
\end{abstract}

Keywords: Exoplanets, $\alpha$ Centauri AB Star System, $\alpha$ Centauri Bb, Sublimation Lifetime

\section{Introduction}

Since the discovery of $51 \mathrm{Peg} \mathrm{Ab}$, the first exoplanet to be identified in orbit about a Sun-like, main sequence star, now some 20 years ago, an additional 1945 exoplanets have been detected within 1230 stellar systems (as of 16 August, 2015). It is now clear that a whole range of system architectures exist, with exoplanets being located as close as 0.006 au (as in the case of Kepler-70b and Kepler-42c) and as far as 3200 au (in the case of HIP 77900b) from their parent stars. Exoplanets have been found in orbit around bona fide single stars, in orbit about both stars within a binary system, and in orbit about the individual stars within a binary system. The available data additionally indicates that planets are extremely common. Current survey data, for example, indicate that $25 \%$ of all Sun-like stars have at least one planet with a mass between 1 and $2 \mathrm{M}_{\text {Earth }}$ and an orbital period between 5 and 100 days.

Within the solar neighborhood, out to a distance of $10 \mathrm{pc}$ from the Sun, there are a total of 321 stars, 20 white dwarfs, 28 brown dwarfs, and an associated 16 planet/planetary systems [1]. The closest such exoplanet, located just $1.35 \mathrm{pc}$ away, is $\alpha$ Cen $\mathrm{Bb}$. The alpha-Centauri triple system is composed of the close binary pair $\alpha$ Cen $\mathrm{AB}$, and the wide binary system composed of $\alpha$ Cen $\mathrm{AB}$ and Proxima Centauri [1]. In terms of physical characteristics $\alpha$ Cen A is a G2 V spectral type star, $\alpha$ Cen B is a K1 V type star, and Proxima Centauri is a M5.5 V spectral type flare star. It has long been expected that planets might well have formed and survived on stable orbits in the $\alpha$ Cen AB binary [1,2], and the first such planet was provisionally detected by Xavier Dumusque and co-workers in 2013 [3]. Orbital stability studies indicate that planets out to distance of some 4 au from $\alpha$ Cen B could well exist, and $\alpha \mathrm{Cen} \mathrm{Bb}$, having an orbital semi-major axis of 0.04 au and an orbital period of just 3.24 days, is perhaps just the first of several terrestrial mass planets orbiting $\alpha$ Cen $\mathrm{B}$ awaiting discovery [4]. The provisional orbit of $\alpha \mathrm{Cen} \mathrm{Bb}$ is determined as being circular, and the minimum mass is set at 1.13 Earth masses.

Alpha Centauri B can presently be categorized as a minimum STIPS (system of tightly packed planets), and being situated so close to its parent star the equilibrium surface temperature of $\alpha \mathrm{Cen} \mathrm{Bb}$ will be in excess of onethousand Kelvin (see below for calculation details). The high surface temperature of $\alpha \mathrm{Cen} \mathrm{Bb}$ dictates that mass loss through sublimation must be taking place, and the question thus arises as to how long can the planet survive? Conversely, 
if the age of the $\alpha$ Cen system can be determined, and the current mass of $\alpha \mathrm{Cen} \mathrm{Bb}$ established, then an estimate of the planets initial formation mass can be made.

Results from the Kepler Mission survey data indicate that of order $5 \%$ of FGK spectral type stars are STIPS, with perhaps as many as $50 \%$ of stars having at least one 0.8 to 2 Earth mass planet with orbital periods smaller than that of Mercury ( $\sim 88$ days) within our own solar system. Volk and Gladman [5] have further suggested that virtually all FGK stars form as STIPS, but subsequent orbital instability, collisions, ejection and destruction interactions result in the rapid clearing-out of the innermost planets. Volk and Gladman argue that the clearing timescale is of order several hundred million years, and accordingly, given a system age much greater than this timescale (see below) we take this result to indicate that $\alpha \mathrm{Cen} \mathrm{Bb}$ has long occupied a stable orbit. This suggests that $\alpha \mathrm{Cen} \mathrm{Bb}$ is either a STIPS survivor, or that it may have formed further outwards from $\alpha$ Cen B and migrated inwards to its currently deduced orbit. Plavchan, Chen and Pohl [6] have investigated the likely outcomes of orbital migration of $\alpha \mathrm{Cen} \mathrm{Bb}$, and find that under a wide range of initial orbital inclinations and starting positions, $\alpha$ $\mathrm{Cen} \mathrm{Bb}$ arrives at something very close to its present orbital radius on a timescale of order 10 to 100 million years. Usefully, as well, for our study to follow, Plavchan, Chen and Pohl find an upper mass limit of 2.7 Earth masses for $\alpha$ Cen $\mathrm{Bb}$, and this strongly argues for a terrestrial planet-like composition. The studies by Volk and Gladman along with that by Plavchan, Chen and Pohl, lead us to suggest that $\alpha$ $\mathrm{Cen} \mathrm{Bb}$ has occupied a stable orbit at or very near to its present location from $\alpha$ Cen $B$ for essentially the entire lifetime of the $\alpha$ Cen AB system.

Estimates for the age of the $\alpha$ Cen $\mathrm{AB}$ system $T_{\text {sys }}$ must be based upon comparing stellar model characteristics against the observed luminosity and the inferred mass of the system's stars, and accordingly estimates have varied from as young as 5 billion to as old as 7 billion years [1]. Most recently, based upon a five-dimensional parameter space study, Bazot, Bourguignon and Christensen-Dalsgaard [7] suggest an age of $6.7 \pm 0.5$ Gyr for $\alpha$ Cen AB. In contrast, based upon a study of rotational velocities, Mamajek and Hillenbrand [8] find a gyro age of $\tau_{\mathrm{gy}}=5.0 \mathrm{Gyr}$ for $\alpha$ Cen A. In the study to follow we adopt an age window of between 5 and 7 Gyr for the system age, and provisionally take 6 billion years as being representative.

\section{The Sublimation Model}

Mass loss through surface material sublimation and the eventual destruction of planets, asteroids and comets have been studied under numerous astrophysical contexts. Jura [9] has examined the destruction of extrasolar asteroid belts during the post main sequence stage of their parent stars evolution; Zubovas, Nayakshin and Markoff [10] have additionally considered the destruction and accretion of asteroids by the super massive black hole located at the center of our galaxy - suggesting that some of the flares recorded for Sgr A* could be due to asteroid as well as planet evaporation, accretion and tidal disruption. On a smaller astrophysical scale, Perez-Becker and Chiang [11] have investigated the evaporation lifetime for the rocky exoplanet detected in orbit around the K-spectral type star KIC 12557548 - finding a disintegration time shorter than $10 \mathrm{Gyr}$. This latter result indicates that it is entirely possible that close-in planets can be fully destroyed, through sublimation mass loss, on a timescale smaller than the main sequence lifetime of the parent star.

The sublimation model to be developed will require the annotation of numerous system parameters. The basic process of sublimation is controlled by the prevailing surface temperature, and this in turn will depend upon the planet's orbital characteristics, the luminosity of the parent star, and the age of the system and the characteristic composition of the planet's surface material. For $\alpha \mathrm{Cen} \mathrm{Bb}$ we adopt a fixed orbital radius of $D=0.04 \mathrm{au}$, and assume the orbital eccentricity is zero at all times. The temperature $T$ of the planet is accordingly determined by the time-dependent luminosity $L(t)$ of $\alpha$ Cen B, and the planet's surface albedo $A$ and emissivity $\mathcal{E}$. Accordingly,

$$
T=278\left(\frac{(1-A) L(t)}{\varepsilon D^{2}}\right)^{1 / 4}
$$

The albedo and emmissivity are assumed constant in this analysis, and we adopt $A=0.3$ and $\varepsilon=0.9$ as characteristic values - the luminosity and orbital radius in equation (2.1) are expressed in solar units and astronomical units respectively. The surface mass loss rate $\sigma(T)$ is determined via Langmir's equation and a suitable, material-specific, phase-equilibrium vapor pressure formulation $p_{\mathrm{v}}$. Accordingly, the rate of change of radius $R$ (for an assumed spherical planet) will be

$$
\frac{d R}{d t}=-\frac{\sigma(T)}{\rho_{B}}
$$

where $\rho_{B}$ is the bulk density of the planet. The vapor pressure term is dependent upon the adopted composition for the planet's surface material. Here we consider just three specific compositional terms - those for iron $(\mathrm{Fe})$, fayalite $\left(\mathrm{Fe}_{2} \mathrm{SiO}_{4}\right)$ and forsterite $\left(\mathrm{Mg}_{2} \mathrm{SiO}_{4}\right)$. In the models considered, the planets have iron cores, and either fayalite or forsterite mantles. The parameterization for the vapor pressure terms are taken directly from the work by van Lieshout, Min and Dominik [12], and using the representation that $p_{\mathrm{v}}=\exp (-$ $\mathrm{A} / \mathrm{T}+\mathrm{B})$, table 1 provides the relevant parameter information.

Table 1. Compositional parameter space. Column 1 indicates the compositional term, columns 2, 3 and 4 indicate the density, mean molecular weight, and evaporation coefficients respectively. Columns 5 and 6 show the parameters used to determine the equilibrium vapor pressure. Units are cgs for all quantities. Data is from [12] and the collected references there in.

\begin{tabular}{llllll}
\hline Composition & $\boldsymbol{\rho}$ & $\boldsymbol{\mu}$ & $\boldsymbol{\alpha}$ & $\mathbf{A}$ & $\mathbf{B}$ \\
\hline Iron & 7.87 & 55.845 & 1.0 & $48354 \pm 1151$ & $29.2 \pm 0.7$ \\
Fayalite & 4.39 & 203.774 & 0.1 & $60377 \pm 1082$ & $37.7 \pm 0.7$ \\
Forsterite & 3.27 & 140.694 & 0.1 & $65308 \pm 3969$ & $34.1 \pm 2.5$ \\
\hline
\end{tabular}


The specific minerals selected for consideration (Table 1) reflect those expected of a differentiated planet with an ironrich core and a silicate-rich mantle. On Earth olivine is the most common silicate material in the upper mantle region, and fayalite and forsterite represent the extreme iron-rich to magnesium-rich ends of the olivine series. Additionally, fayalite and forsterite minerals have been identified in Moon rocks, meteorite samples (both from Mars and the main belt asteroid belt region) and within cometary refractory materials, indicative of a wide dispersion within the solar system. Olivine minerals have also been identified, using infrared reflectance spectroscopy techniques, in the dust clouds surrounding newly forming protostars, suggestive of the likelihood that such minerals will be common to all terrestrial exoplanet. Other mantle materials might reasonably have been included in our analysis, but the vapor pressure data, as found within the available literature [see e.g., 9, 11, 12], reveals that, at a given temperature, the sublimation rate of fayalite is very similar to that of silicon monoxide ( $\mathrm{SiO})$, and that for forsterite is similar to those of enstatite $\left(\mathrm{MgSiO}_{3}\right)$ and quartz $\left(\mathrm{SiO}_{2}\right)$. Additionally, for a given temperature, the sublimation rates of fayalite and iron are about the same, with both being some three-orders of magnitude higher than that for forsterite. Accordingly, we adopt the stance that forsterite and fayalite will be common in terrestrial exoplanets, and that these two minerals represent the extremes in the sublimation characteristics of typical silicate mantel materials.

A two component, differentiated terrestrial planet model is adopted in this study; the model assumes an iron core of radius $R_{\mathrm{C}}$ and density $\rho_{\mathrm{c}}$, surrounded by a mantle of density $\rho_{\mathrm{m}}$. The planet is taken to have an initial radius $R_{0}$, and this is in turn linked to the bulk density via the formula given by Marcy et al [13], with

$$
\rho_{B}=2.32+3.19\left(\frac{R_{0}}{R_{E}}\right)
$$

where $R_{E}=6371 \mathrm{~km}$ is the Earth's radius. With the initial bulk density determined the initial mass is set according to the standard density relationship: $M_{0}=(4 \pi / 3) R_{0}{ }^{3} \rho_{B}$. The initial core radius is now determined and evaluated via the formula

$$
R_{C}=R_{0}\left(\frac{\rho_{B}-\rho_{m}}{\rho_{C}-\rho_{m}}\right)^{1 / 3}
$$

with the core mass following as $M_{C}=(4 \pi / 3) R_{C}{ }^{3} \rho_{C}$. The initial model is now fully described and is determined by the choice of $R_{0}$. The sublimation sequence is evaluated according to the time dependent radius $R(t)$, as determined through the solution to equation (2.2), with either $R(t)>R_{C}$ and $R(t) \leq R_{C}$. In this manner,

If $R(t)>R_{C}$ then:

$$
\rho_{B}=x^{3}\left(\rho_{C}-\rho_{\mathrm{m}}\right)+\rho_{m}, \text { where } x=R_{C} / R(t), \text { and }
$$

$$
M(t)=M_{C}+(4 \pi / 3) \rho_{m}\left[1-x^{3}\right] R(t)^{3}
$$

If $R(t) \leq R_{C}$ then:

$$
\begin{gathered}
\rho_{B}=\rho_{C}=\rho(\mathrm{Fe}) \text {, and } \\
M(t)=(4 \pi / 3) R(t)^{3} \rho_{c} .
\end{gathered}
$$

By way of example numbers, for a model Earth with $R_{0}=$ $1.0 R_{E}$ having an iron core with $\rho_{C}=7.87 \mathrm{~g} / \mathrm{cm}^{3}$, and a fayalite mantle with $\rho_{m}=4.39 \mathrm{~g} / \mathrm{cm}^{3}$, we have $\rho_{B}=5.51$ $\mathrm{g} / \mathrm{cm}^{3}, R_{C} / R_{E}=0.69, M_{0}=5.97 \times 10^{27} \mathrm{~g}$, and $M_{C}=2.80 \times$ $10^{27} \mathrm{~g}$ (which gives $M_{C} / M_{0}=0.47$ ). At this stage our planet models do not include the effects of bulk material compression, or allow for material phase changes due to the presence of an internal temperature gradient (see, however, Section 4 below). Likewise, we do not, at this stage, consider any blanketing effects that might be caused by an overlying atmosphere.

\section{System Evolution}

The equations describing planetary mass loss via sublimation, as presented above, have been combined with an evolutionary stellar model for $\alpha$ Cen B. For the latter we have used a 0.934 solar mass model, having a metal abundance of $Z=0.03$, obtained from the EZ-web server which provides the user with an evolutionary sequence, based upon the Eggleton Code [14]. The time sequence takes the star from the zero age main sequence to the early white dwarf phase. The main sequence lifetime for $\alpha$ Cen B is found to be $T_{m s}=16 \mathrm{Gyr}[1,14]$, and from the first 10 billion years of model data (which comfortably brackets the various age estimates for the $\alpha$ Cen $A B$ ) we have constructed a $4^{\text {th }}$ order polynomial least-squares fit to the model luminosity versus age (figure 1).

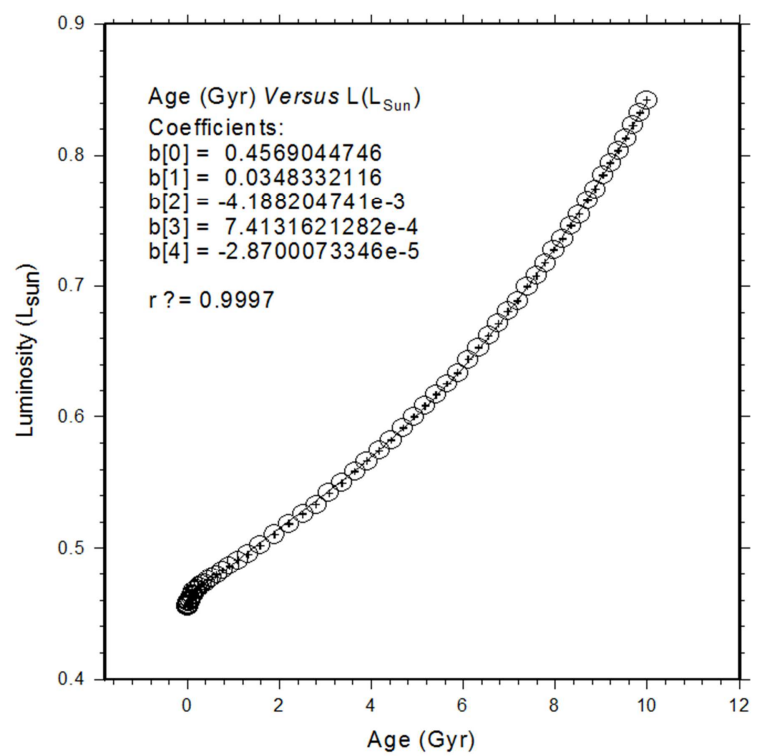

Figure 1. Luminosity (in solar units) versus age (in Gyr) for a 0.934 solar mass model. The coefficients to the $4^{\text {th }}$-order polynomial least-squares fit to the model data points are shown to the upper left of the diagram. Model data from the EZ-web server [14]. 
Table 2. Sublimation lifetimes for pure composition (forsterite, fayalite and iron) planets. Columns 2 and 3 provide the initial radius and mass in Earth units. Column 4 is the final mass after a time $T_{\text {sub }}$ (as given in column 5). The final column indicates the time at which the planet's mass drops below 1 $M_{\text {Earth }}$

\begin{tabular}{llllll}
\hline Composition & $\boldsymbol{R}_{\mathbf{0}} / \boldsymbol{R}_{\boldsymbol{E}}$ & $\boldsymbol{M}_{\mathbf{0}} / \boldsymbol{M}_{\boldsymbol{E}}$ & $\boldsymbol{M}_{f} / \boldsymbol{M}_{\boldsymbol{E}}$ & $\boldsymbol{T}_{\text {sub }}(\mathbf{G y r})$ & $\boldsymbol{T}_{\mathbf{1}}(\mathbf{G y r})$ \\
\hline Forsterite & 0.75 & 0.250 & 0.249 & 10 & -- \\
Fayalite & 0.75 & 0.34 & $<$ Moon & 8.6 & --- \\
Iron & 0.75 & 0.60 & $<$ Moon & 6.74 & --- \\
Forsterite & 1.00 & 0.593 & 0.591 & 10 & --- \\
Fayalite & 1.00 & 0.80 & $<$ Moon & 9.17 & --- \\
Iron & 1.00 & 1.43 & $<$ Moon & 7.44 & 3.72 \\
Forsterite & 1.25 & 1.158 & 1.155 & 10 & --- \\
Fayalite & 1.25 & 1.56 & $<$ Moon & 9.59 & 7.03 \\
Iron & 1.25 & 2.79 & $<$ Moon & 7.95 & 5.95 \\
Forsterite & 1.50 & 2.001 & 1.996 & 10 & --- \\
Fayalite & 1.50 & 2.69 & $<$ Moon & 9.88 & 8.35 \\
Iron & 1.50 & 4.82 & $<$ Moon & 8.33 & 6.96 \\
Forsterite & 1.75 & 3.179 & 3.171 & 10 & --- \\
Fayalite & 1.75 & 4.27 & 0.04 & 10 & 9.01 \\
Iron & 1.75 & 7.65 & $<$ Moon & 8.65 & 7.59 \\
\hline
\end{tabular}

Table 2 provides a series of initial, comparative, calculations for homogeneous planet models composed of forsterite, fayalite and iron. In each case the orbital radius is taken as $D=0.04 \mathrm{au}$, and the sequence is run until $t=10 \mathrm{Gyr}$, or until the planet mass drops below that of Earth's Moon, $\mathrm{M}_{\text {Moon }}=0.0123 \mathrm{M}_{\text {Earth }}$, at a time corresponding to $T_{\text {sub }}$. The Moon's mass is not fundamental, as such, but it is a convenient mass below which it is taken that the planet has effectively evaporated. Also shown in column 6 of table 2 is the time $T_{1}$ at which the planet's mass drops below that of the Earth. Again, this latter mass, and its associated age, is not fundamental, as such, but reflects the fact that the estimated minimum mass of $\alpha \mathrm{Cen} \mathrm{Bb}$ is determined as $1.13 \mathrm{M}_{\text {Earth, }}$. Effectively, we are interested in looking at planetary models that fit within the mass range $1.1<M(t) / M_{\text {Earth }}<2.7$ after a time $t=T_{s y s}<T_{1}$ has passed. While our primary interest lies with the results from differentiated planet models (as discussed below), we find with the aid of figure 2 that a super-Mercury, solid iron planet with an initial radius of about $1.5 R_{\text {Earth }}$ (that is, with an initial mass of about $5 M_{\text {Earth }}$ ) will have been reduced to a mass comparable to the minimum set for $\alpha \mathrm{Cen} \mathrm{Bb}$ (i.e., $\sim 1 M_{\text {Earth }}$ ) when the system is $\sim 7$ billion years old. If the system age is as small as $5 \mathrm{Gyr}$, then an iron planet of initial mass $\sim 2$ Earth masses is required in order to satisfy the minimum mass value for $\alpha$ Cen $\mathrm{Bb}$. A monolithic, silicate planet composed of fayalite having an initial radius of about $1.3 R_{\text {Earth }}$ (mass $\sim 1.8 M_{\text {Earth }}$ ) will have a mass comparable to that of the minimum deduced for $\alpha \mathrm{Cen} \mathrm{Bb}$ after a time $\sim 7$ billion years (see figure 3 ). At the lower system age estimate of $5 \mathrm{Gyr}$, the initial mass required of a pure silicate planet of fayalite to satisfy the lower mass limit for $\alpha \mathrm{Cen} \mathrm{Bb}$ is about $1.4 M_{\text {Earth }}$ (initial radius $\left.\sim 1.2 R_{\text {Earth }}\right)$. Since the sublimation rate for forsterite is so low, the initial mass of a monolithic silicate planet composed of forsterite need only be comparable to the actual mass and system age for $\alpha \mathrm{Cen} \mathrm{Bb}$ (what ever those values actually turn out to be - see discussion below). Looking to the upper mass range limit of $2.7 \mathrm{M}_{\text {Earth }}$ to $\alpha \mathrm{Cen} \mathrm{Bb}$, the initial mass for a super-Mercury planet would have to be of order 4.4 $\mathrm{M}_{\text {Earth }}$ (see figure 2); for a pure silicate planet composed of fayalite the required initial mass range would need to fall between 3.0 and 3.8 Earth masses (see figure 3).

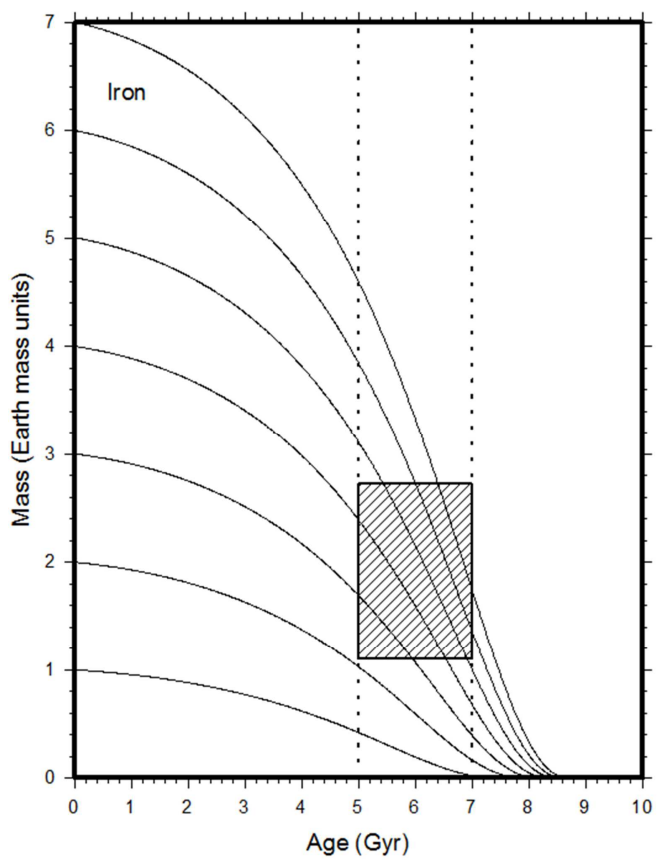

Figure 2. Evolutionary tracks for super-Mercury, iron planets. The orbital distance is fixed at $D=0.04 \mathrm{au}$, and the time sequence follows the luminosity evolution of $\alpha$ Cen B. The cross-hatched box indicates the estimated system age for $\alpha$ Cen $B$ and the estimated mass range for $\alpha$ Cen $\mathrm{Bb}$.

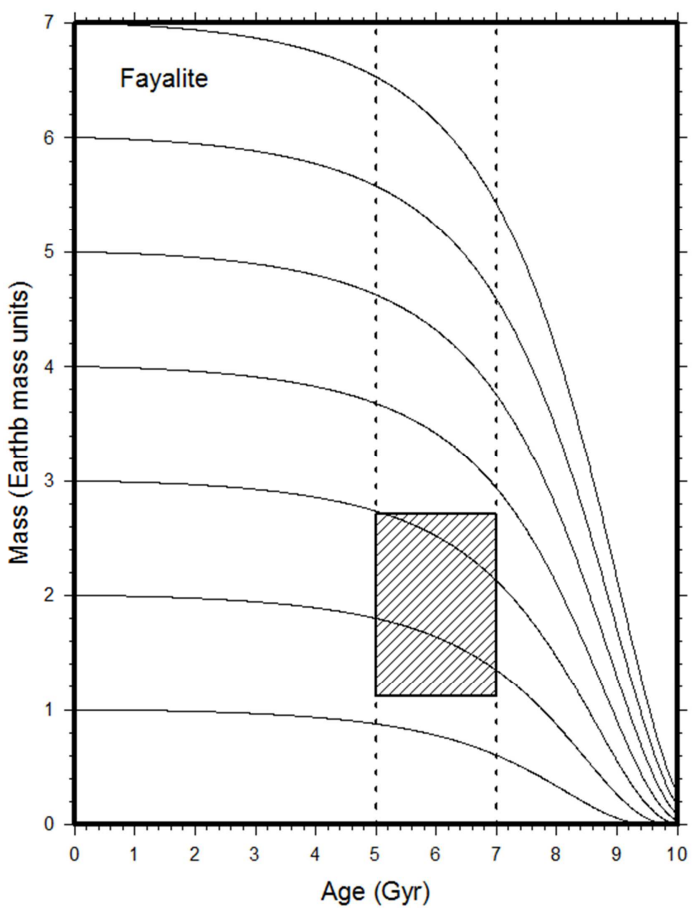

Figure 3. Evolutionary tracks for monolithic fayalite planets. The orbital distance is fixed at $D=0.04 \mathrm{au}$, and the time sequence follows the luminosity evolution of $\alpha$ Cen $B$. The cross-hatched box indicates the estimated system age for $\alpha$ Cen $B$ and the estimated mass range for $\alpha$ Cen $\mathrm{Bb}$. 
Homogeneous pure-iron, or super-Mercury, planets represent the smallest sized planets (for a given mass) that might potentially exist; pure-silicate planets always being somewhat larger because of their lower intrinsic densities. With respect to the single composition models we find that $\alpha$ $\mathrm{Cen} \mathrm{Bb}$ must have an initial mass in the range 1.4 and 5 $M_{\text {Earth }}$ - the former mass corresponding to a pure silicate planet of age $5 \mathrm{Gyr}$, and the latter to a super-Mercury planet of age 7 Gyr. To further refine our initial mass estimates for $\alpha$ Cen $\mathrm{Bb}$, we next investigate the properties and evolutionary characteristics of two-component, core-mantle planet models.

Figure 4 shows the evolutionary tracks for the mass of composite iron-core, fayalite-mantle planets. The results are complementary to those derived for the pure-iron and puresilicate models. The dashed line in figure 4 indicates the time at which the iron core is first revealed - that is the time for the mantle to fully sublimate - and it would appear that a super-Mercury-like model for $\alpha$ Cen Bb can be ruled-out. While (from figure 2) a pure-iron model planet of initial mass $5 \mathrm{M}_{\text {Earth }}$ will attain a minimum $\alpha$ Cen $\mathrm{Bb}$ mass of $1.1 \mathrm{M}_{\text {Earth }}$ after 7 billion years, the same initial mass iron-fayalite composite model only achieves this minimum mass limit after some 9.5 billion years. Composite iron-core and forsterite-mantle models take even longer to achieve core exposure - indeed, they only do so on a timescale greater than the main sequence lifetime of $\alpha$ Cen B. Typically, for the forsterite-mantle models we find only of order 0.002 $\mathrm{M}_{\text {Earth }} \sim 1 / 6^{\text {th }} \mathrm{M}_{\mathrm{Moon}}$ of mantle material being lost during the first 10 billion years of $\alpha$ Cen $B$ evolution.

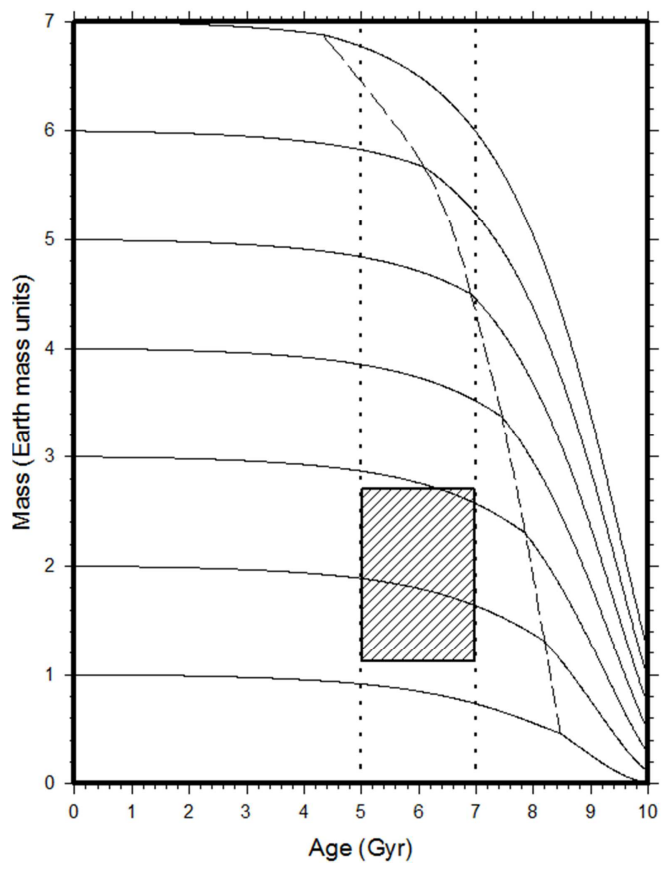

Figure 4. Evolutionary tracks for composite iron-core, fayalite-mantle planets. The orbital distance is fixed at $D=0.04 \mathrm{au}$, and the time sequence follows the luminosity evolution of $\alpha$ Cen $B$. The cross-hatched box indicates the estimated system age for $\alpha$ Cen $B$ and estimated mass range for $\alpha$ Cen $B b$. The dashed line indicates the time at which the iron core is first exposed at the surface.

\section{Discussion}

The composite iron-core, fayalite-mantle planetary models (figure 4) indicate that for a 6 billion year system age, the initial mass associated with $\alpha \mathrm{Cen} \mathrm{Bb}$ will reside somewhere in the range 1.3 to $2.9 \mathrm{M}_{\text {Earth }}$, indicating that of order 0.2 $\mathrm{M}_{\text {Earth }}$ of mantle material has been lost through sublimation. The model further suggests that $\alpha \mathrm{Cen} \mathrm{Bb}$ will begin to transition into a super-Mercury planet (i.e., have no silicate mantle) within the next 2 billion years. We also find that the complete sublimation destruction of $\alpha \mathrm{Cen} \mathrm{Bb}$ will occur within the next 6 billion years, on a timescale well within the 16 billion year main sequence lifetime of $\alpha$ Cen B.

The iron-core, forsterite-mantle planetary models indicate that the initial mass of $\alpha \mathrm{Cen} \mathrm{Bb}$ is essentially that which is observed now (or, at least, the mass to be confirmed by future observations), with of order $0.001 \mathrm{M}_{\text {Earth }}\left(6 \times 10^{21} \mathrm{~kg} \sim 7\right.$ times the mass of dwarf planet Ceres) of mantle material being lost via sublimation over the past 6 billion years. Running the evolutionary sequence through to end of the entire $16 \mathrm{Gyr}$ main sequence life time of $\alpha$ Cen $B$ reveals that only $\sim 0.1$ $\mathrm{M}_{\text {Earth }}$ (or $\sim 2$ times the mass of planet Mercury) of mantle material is lost via sublimation. Even if the mantle of $\alpha$ Cen $\mathrm{Bb}$ is composed of material with an extremely low sublimation rate, such as that for forsterite, the ultimate destruction of the planet will occur shortly after $\alpha$ Cen B enters its post main sequence phase $[1,15]$, since at that time the radius of $\alpha$ Cen $\mathrm{B}$ will expand beyond the present orbital radius limit of 0.04 au $\left(=9 \mathrm{R}_{\odot}\right)$. Indeed, once entrained within the expanding red giant envelope of $\alpha$ Cen $B$, the planet's lifetime against destruction will be of order several hundreds of years $[1,15]$. The ultimate destruction epoch for $\alpha$ Cen Bb is set, by the $\alpha$ Cen B stellar model, at a system age of about $16.5 \mathrm{Gyr}$, or some $10 \mathrm{Gyr}$ from the present.

The presently available radial velocity data indicate that $\alpha$ Cen B has no planets more massive that $1.5 \mathrm{M}_{\text {Jupiter }}$ orbiting closer than 2 au $[1,16]$; a result that leaves plenty of room for the potential detection of terrestrial mass planets (here taken to be objects less massive than $\sim 10 \mathrm{M}_{\text {Earth }} \approx 0.006$ $\mathrm{M}_{\text {Jupiter }}$ ) in the future. It is presently unclear if super-Earth planets, with masses several times that of the Earth, can form within a relatively close binary system such as $\alpha$ Cen $A B$, and recent planet formation models suggest that only objects in the mass range $\sim 1$ to $2 \mathrm{M}_{\text {Earth }}$ are likely to accrete within the zone 1.5 to 0.5 au from $\alpha$ Cen B $[17,18,19]$. Planets in this orbital radius range will not suffer any significant mantle sublimation mass loss effects over the entire main sequence lifetime of $\alpha$ Cen B. Combining the results from the planet formation models with the dynamical lifetime limit set by Plavchan, Chen and Pohl [6], it would not seem unreasonable to adopt an initial mass of about $2 \mathrm{M}_{\text {Earth }}$ for $\alpha$ Cen Bb. Since the mass of a planet derived from radial velocity data [3] increases as the inverse sine of the orbital inclination, so a mass estimate of $2 \mathrm{M}_{\text {Earth }}$ for $\alpha$ Cen Bb suggests an orbital inclination of about 33 degrees to our line of sight at the present epoch. Additionally, since the orbital plane of $\alpha$ Cen $\mathrm{AB}$ is inclined by 79.2 degrees to our line of sight on the sky 
[1], this suggests a relatively large, $\sim 45$ degrees, off-set angle for the orbital plane of $\alpha \mathrm{Cen} \mathrm{Bb}-\mathrm{a}$ result that is consistent with the non-detection of planetary transits [4]. Interestingly, the numerical study by Quintana et al. [19] on planet formation within the $\alpha$ Cen $\mathrm{AB}$ system indicates that for high disk inclinations one or two large, that is Earth mass, planets tend to form at an orbital radius of between 1.0 and $0.5 \mathrm{au}$, with multiple sub-Earth mass planets forming in the region between 1 and 2 au. At 0.5 au, a $1 \mathrm{M}_{\text {Earth }}$ planet in orbit around $\alpha$ Cen B would not be detectable with currently available radial velocity techniques (and this is especially so given the highly complex deconvolution that is required to extract a potential planetary signal from the stellar rotation and chromospheric activity - noise - associated with $\alpha$ Cen B [3]). However, a high orbital inclination is consistent with the early migration inwards via the Kozai mechanism, as described by Plavchan, Chen and Pohl [6], and the presentday orbital location of $\alpha \mathrm{Cen} \mathrm{Bb}$.

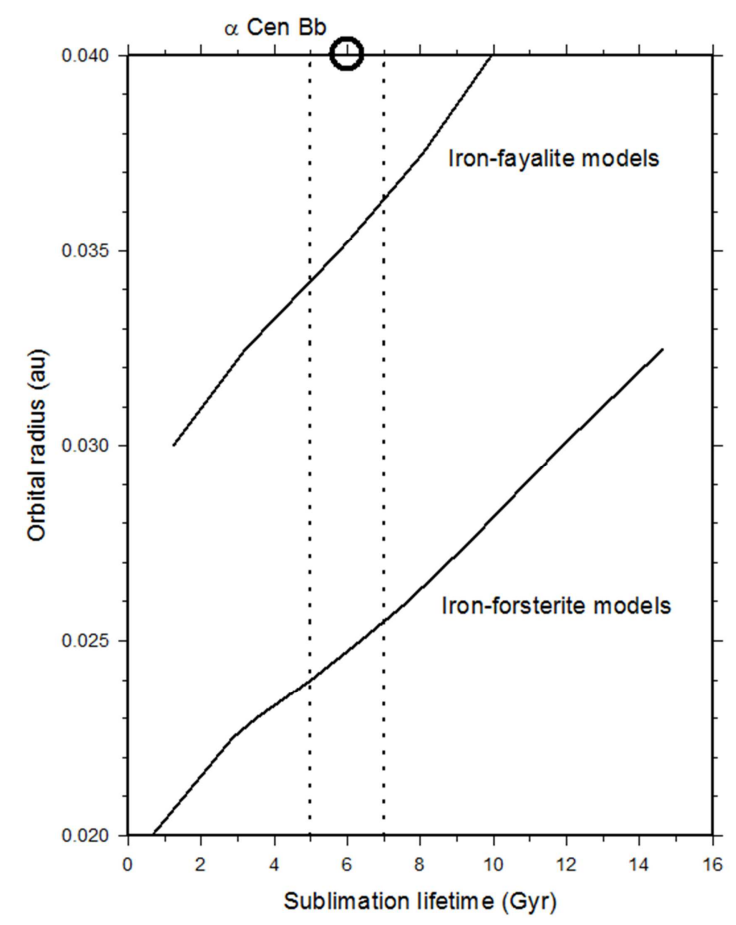

Figure 5. Lifetime against destruction by sublimation for an initial $1 M_{\text {Earth }}$ mass planet located interior to the orbit of $\alpha \mathrm{Cen} B b$. The vertical dotted lines correspond to the maximum and minimum $\alpha C e n$ AB system age limits. The slope turnover in the smallest orbit, most rapidly sublimated models (particularly evident in the iron-forsterite models), relates to the prompt exposure and then rapid evaporation of the iron-core.

What about the possibility of planets interior to the orbit of $\alpha$ Cen $\mathrm{Bb}$ ? The existence, past and possible present, of subEarth mass planets interior to the orbit of $\alpha \mathrm{Cen} \mathrm{Bb}$ is entirely consistent within the STIPS framework [4], but the lifetimes against sublimation destruction will consequently decrease because of the higher surface temperatures that such planets will experience. Figure 5 shows the variation of the lifetime against destruction by sublimation for a $1 \mathrm{M}_{\text {Earth }}$ planet (core-mantle models) for a selection of orbital radii between 0.04 and $0.02 \mathrm{au}$. The figure indicates that a $1 \mathrm{M}_{\text {Earth }}$ planet, having an iron-core and a fayalite-mantle, just 0.006 au closer (orbital radius of $0.034 \mathrm{au}$ ) to $\alpha$ Cen B than $\alpha$ Cen $\mathrm{Bb}$ will have fully sublimated on a timescale shorter than 5 billion years. Likewise an Earth mass planet, having an ironcore and a forsterite-mantle, will have fully sublimated on a timescale shorter than 5 billion years if located on an orbit closer than 0.024 au to $\alpha$ Cen B.

All that has been presented in the discussion above is admittedly speculation; but it is speculation consistent within the parameter space of both the observational uncertainties and the present-day status of theoretical models on planet formation in the $\alpha$ Cen $\mathrm{AB}$ system. At this stage we can only conclude that there are many details, both observational as well as theoretical, that will need to be resolved before any clear idea of the true structure of the $\alpha$ Cen B planetary system, and where it sits within the great panoply of exoplanet architectures [20, 21, 22], becomes available. Clearly, the first step for the future is to confirm [23] the analysis of Dumusque et al. [3], and then further quantify any planetary system that might be associated with $\alpha$ Cen B (and eventually $\alpha$ Cen A, and Proxima Centauri $[1,24,25])$. Future work in relation to the sublimation model presented in Section 2 will focus on incorporating more realistic planetary models into the analysis. Such models will include details concerning bulk composition, material compression and appropriate equations of state for determining planetary mass and radius relationships - see, e.g., Zeng and Seager [26] and Grasset, Schneider and Sotin [27]. Additional future work will also consider sublimation effects in other close-in exoplanetary systems (e.g., Kepler-70b and Kepler -42c), along with dust, Vulcanoid [28], asteroid and cometary cloud sublimation scenarios in both young and evolved stellar systems [9, 12, 29, 30].

\section{References}

[1] Beech, M. Alpha Centauri: unveiling the secrets of our nearest stellar neighbor. Springer, New York, 2015.

[2] Wiegert, P., and Holman, M. J. 1997. The stability of planets in the alpha Centauri system. Astron. J. 113, 1445.

[3] Dumusque, X., et al. 2012. An Earth mass planet orbiting alpha Centauri B. Nature, 491, 207.

[4] Brice-Olivier, D., et al. 2015. Hubble Space Telescope search for the transit of the Earth-mass exoplanet Alpha Centauri Bb. http://arxiv.org/abs/1503.07528v1.

[5] Volk, K., and Gladman, B. 2015. Consolidating and crushing exoplanets: did it happen here? http//arxiv.org/abs/1502.065581v1.

[6] Plavchan, P., Chen, Xi, and Pohl, G. 2015. What is the mass of $\alpha$ Cen Bb? Ap. J. 805, id.174.

[7] Bazot, M., Bourguignon, S., and Christensen-Dalsgaard, J. 2008. Estimation of stellar parameters using Monte Carlo Markov-chains. Mem. Soc. Astron. Italiana. 79, 660.

[8] Mamajek, E. E., and Hillenbrand, L. A. 2008. Improved age estimates for solar-type dwarfs using activity-rotation diagnostics. Ap. J. 687, 1264. 
[9] Jura, M. 2008. Pollution of single white dwarfs by accretion of many small asteroids. Ap. J. 135, 1785.

[10] Zubovas, K., Nayakshin S., and Markoff, S. 2011. Sgr A* flares: tidal disruption of asteroids and planets? http://arxiv.org/abs/1110.6872v1.

[11] Perez-Becker, D., and Chiang, E. 2013. Catastrophic evaporation of rocky planets. http://arxiv.org/abs/1302.2147v3.

[12] Van Lieshout, R., Min, M., and Dominik, C. 2014. Dusty tails of evaporating exoplanets 1. Constraints on the dust composition. Astron. Astrophys. 572, id.A76.

[13] Marcy, G. W., et al. 2014. Occurrence and core-envelope structure of 1-4xEarth-size planets around Sun-like stars. PNAS. 111, 12655.

[14] Townsend, R. 2015. http://www.astro.wisc.edu/ townsend/static.php?ref=ez-web.

[15] Beech, M. 2011. The far distant future of Alpha Centauri. JBIS. 64, 387.

[16] Endl, M., Kurster, M., Els, S., Hatzes, A. P., and Cochran, W. D. 2001. The planet search program at the ESO Coude Echelle spectrometer II. The alpha Centauri system: Limits for planetary companions. Astron. Astrophys. 374, 675.

[17] Guedes, J. M. et al. 2008. Formation and detectability of terrestrial planets around $\alpha$ Centauri B. Ap. J. 679, 1582.

[18] Thebauld, P., Marzari, F., and Scholl, H. 2009. Planet formation in the habitability zone of alpha Centauri B. MNRAS. 393, L21.

[19] Quintana, E. V., Lissauer, J. J., Chambers, J. E., and Duncan, M. J. 2002. Terrestrial planet formation in the $\alpha$ Centauri system. Ap. J. 576, 982.

[20] Ford, E. D. 2014. Architectures of planetary systems and implications for their formation. PNAS. 111, 12616.
[21] $\mathrm{Pu}, \mathrm{B}$. and $\mathrm{Wu}, \mathrm{Y}$. 2015. Spacing of Kepler planets: sculpting by dynamical instability. http://arxiv.org/abs/1502.05449v1.

[22] Lissauer, J. J., Dawson, R. I., and Tremaine, S. 2014. Advances in exoplanet science from Kepler. Nature, 513, 336.

[23] Hatzes, A. P. 2013. The radial velocity detection of Earth mass planet in the presence of activity noise: the case of $\alpha \mathrm{Cen} \mathrm{Bb}$. Ap. J. 770, id.133.

[24] Bergmann, C., Endl, M., Hearnshaw, J., Wittenmyer, R., and Wright, D. 2015. Searching for Earth-mass planets around $\alpha$ Centauri: precise radial velocities from contaminated spectra. Int. J. Astrobiology. 14, 173.

[25] Endl, M., and Kuster, M. 2008. Towards detection of terrestrial planets in the habitable zone of our closest neighbor: Proxima Centauri. Astron. Astrophys. 488, 1149.

[26] Zeng, L., and Seager, S. 2008. A computational tool to interpret the bulk composition of solid exoplanets based on mass and radius measurements. Pub. Astron. Soc. Pacific. 120, 983.

[27] Grasset, O., Schneider, J., and Sotin, C. 2009. A study of the accuracy of mass-radius relationships for silicate-rich and icerich planets up to 100 Earth masses. Ap. J. 693, 722.

[28] Beech, M., and Peltier, L. 2015. Vulcanoid asteroids and Sungrazing comets: past encounters and possible outcomes. American Journal of Astronomy and Astrophysics, 3(2), 26.

[29] Lin, H., and Loeb, A. 2014. Finding rocky asteroids around white dwarfs by their periodic thermal emission. Ap. J. Lett. 793, id.L43.

[30] Poteet, C. A. et al. 2011. A Spitzer infrared spectrograph detection of crystalline silicates in a protostellar envelope. Ap. J. Lett. 733, id.L32. 